Original

\title{
Prevalencia de endoparásitos gastrointestinales y ectoparásitos en serpientes ex situ en Barranquilla, Colombia
}

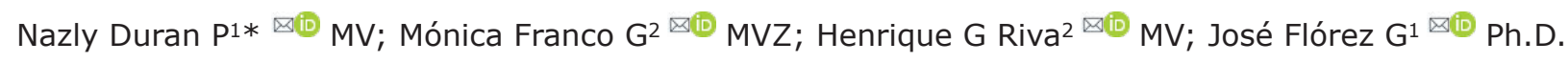

1Universidad de Pamplona, Facultad de Ciencias Agrarias, Programa de Medicina Veterinaria, Km 1 vía Bucaramanga Ciudad Universitaria, Pamplona, Colombia.

²Fundación Botánica y Zoológica de Barranquilla, Departamento de Veterinaria, Calle 77\#68-40, Barranquilla, Colombia.

*Correspondencia: mvduranazly@outlook.es

Recibido: Abril 2019; Aceptado: Agosto 2019; Publicado: Enero 2020.

\section{RESUMEN}

Objetivo. Identificar la prevalencia de endoparásitos y ectoparásitos en serpientes ex situ en la Fundación Botánica y Zoológica de Barranquilla "Barranquilla Zoo". Materiales y métodos. Para esto se analizaron muestras de materia fecal y ectoparásitos de 2 colúbridos (Leptophis ahaetulla y Spilotes pullatus), 2 dipsadinos (Oxybelys aeneus y Thamnodynastes paraguanae) y 13 boidos (9 individuos de la especie Boa constrictor, 2 de Epicrates maurus y 2 de Eunectes murinus) utilizando los métodos de flotación en solución saturada de azúcar y observación directa. Resultados. Los resultados obtenidos mostraron que el 65\% (11/17) de los animales evaluados fueron positivos a la presencia de parásitos, de los cuales el 91\% (10/11) de los ejemplares pertenece a la familia Boidae presentando en su mayoría nematodos, mientras que en los dipsadinos se observaron principalmente protozoarios y los colúbridos resultaron negativos. Finamente como ectoparásitos se identificaron 2 especies de garrapatas (Amblyomma dissimile y Ornothodoros turicata) halladas en ejemplares de Boa constrictor, Epicrates maurus y Spilotes pullatus. Conclusiones. La identificación de las formas parasitarias en este trabajo representa una contribución para el conocimiento de los parásitos en serpientes ex situ en Colombia. Provee información para desarrollar medidas profilácticas y permite comparar los datos sobre las formas parasitarias encontradas con muestreos de estas especies en vida silvestre y bajo cuidados humanos.

Palabras clave: Argasidae; Boidae; Colubridae; Dipsadinae; Ixodidae; Parasitología (Fuente: MeSH, DeCS).

\section{ABSTRACT}

Objective. Identify the prevalence of internal and external parasites in ex situ snakes at the Fundación Botánica y Zoológica de Barranquilla "Barranquilla Zoo". Materials and methods. Stool samples and ectoparasites of 2 colubrids (Leptophis ahaetulla and Spilotes pullatus), 2 diapsids (Oxybelys aeneus and Thamnodynastes paraguanae) and 13 boids (9 individuals of Boa constrictor, 2 of Epicrates maurus and 2 Eunectes murinus) were analyzed using flotation method in saturated sugar solution and direct observation. Results. The results showed that $65 \%(11 / 17)$ of the population was positive to the presence of parasites. In relation to the positive specimens, $91 \%$ (10/11) belong to the Boidae

Como citar (Vancouver).

Duran PN, Franco GM, Riva HG, Flórez GJ. Prevalencia de endoparásitos gastrointestinales y ectoparásitos en serpientes ex situ en Barranquilla, Colombia. Rev MVZ Cordoba. 2020; 25(1):e1537. https://doi.org/10.21897/rmvz.1537 CC) 1 (C) (los) autor (es), Revista MVZ Córdoba 2020. Este artículo se distribuye bajo los términos de la licencia internacional Creative Commons Attribution BY NC SA comercial, siempre y cuando den crédito y licencien sus nuevas creaciones bajo las mismas condiciones. 
family, presenting mostly nematodes, in the dipsadid's samples mainly protozoans were observed and the colubrid's samples were negative. Regarding ectoparasites, 2 species of ticks (Amblyomma dissimile and Ornothodoros turicata) were found in specimens of Boa constrictor, Epicrates maurus and Spilotes pullatus. Conclusions. The identification of parasitic forms in this paper represents a contribution to the knowledge of parasites in snakes ex situ in Colombia. It provides information to develop prophylactic measures against these pathological agents and also allows comparing the data about the parasitic forms found with other researches in the wild and in captivity.

Keywords: Argasidae; Boidae; Colubridae; Dipsadinae; Ixodidae; Parasitology (Source: MeSH, DeCS).

\section{INTRODUCCIÓN}

Los zoológicos son instituciones que poseen diversas especies de animales bajo sus cuidados, además incorporan planes educativos de conservación dentro de su misión y ofrecen experiencias a sus visitantes con el objetivo de involucrarlos en temas relacionados con la perdida de la biodiversidad (1); para esto, el manejo de animales ex situ plantea serios desafíos que van desde consideraciones de bienestar animal, requisitos de espacio, habilidades humanas y atención veterinaria (2) ya que el vivir en un ambiente controlado no los deja exentos de presentar parasitosis o cualquier otra alteración en su salud.

Las serpientes in situ y ex situ presentan ectoparásitos y endoparásitos, sin embargo, rara vez son estudiados y por ende poco conocidos. Además de lo anterior, suele examinarse solo un individuo o pequeñas poblaciones. Sin embargo, algunas publicaciones describen nematodos como Dracunculus brasiliensis encontrado en Eunectes murinus (Anaconda) (3), oxiúridos en Boa constrictor y Python regius (4), Cruzia sp., Kalicephalus subulatus, Styphlodora horridum (5) Eimeria spp., Capillaria spp., Sarcocystis spp., Kapsulotaenia spp. (6) Rhabdias spp., Porocephalus spp. en Epicrates cenchria (7) y garrapatas como Rhipicephalus sp., Aponomma sp. y Amblyomma sp. (8).

Los reportes sobre prevalencia de endoparásitos gastrointestinales y ectoparásitos en serpientes en Colombia son escasos, en la revisión de literatura, solo fueron encontrados dos estudios $(9,10)$, que describen el hallazgo de Amblyomma dissimile en dos ejemplares de Boa constrictor y un ejemplar de Spilotes pullatus, respectivamente. Conociendo la importancia de ampliar el conocimiento sobre parásitos en serpientes se llevó a cabo el presente estudio con el objetivo de identificar la prevalencia de endoparásitos gastrointestinales y ectoparásitos en las serpientes bajo cuidado humano en la Fundación Botánica y Zoológica de Barranquilla (FUNDAZOO).

\section{MATERIALES Y MÉTODOS}

Área de Estudio. El estudio se realizó en el herpetario de FUNDAZOO ubicado en la ciudad de Barranquilla - Colombia. Condiciones geoclimáticas: Temperatura promedio mínima de $25^{\circ} \mathrm{C}$ y máxima de $33.5^{\circ} \mathrm{C}$, lluvias que oscilan entre los $70-178 \mathrm{~mm} / \mathrm{mes}$ y humedad relativa de $84 \%$. Coordenadas: $11^{\circ} 00^{\prime} 38^{\prime \prime} \mathrm{N}$ 7447'49"O (IDEAM Colombia).

Animales de estudio. El estudio se realizó durante 12 semanas con las especies de serpientes listadas en la tabla 1. Dos de los 9 individuos de $B$. constrictor se encontraban en el mismo terrario y los demás individuos estaban ubicados en terrarios individuales.

Tabla 1. Especies de serpientes en la colección de FUNDAZOO.

\begin{tabular}{|c|c|c|c|}
\hline $\begin{array}{l}\text { Nombre } \\
\text { común }\end{array}$ & Nombre científico & $\begin{array}{l}\text { Número de } \\
\text { individuos }\end{array}$ & $\begin{array}{l}\text { Peso corporal } \\
\quad(\text { sexo }) *\end{array}$ \\
\hline Boa & Boa constrictor & 9 & $\begin{array}{c}4 \mathrm{~kg}(\mathrm{M}) ; \\
2.6 \mathrm{~kg}(\mathrm{M}) ; \\
2,3 \mathrm{~kg}(\mathrm{M}) ; \\
2.8 \mathrm{~kg}(\mathrm{H}) ; \\
7.7 \mathrm{~kg}(\mathrm{H}) ; \\
4.2 \mathrm{~kg}(\mathrm{H}) ; \\
2 \mathrm{~kg}(\mathrm{H}) ; \\
2 \mathrm{~kg}(\mathrm{H}) ; \\
2.9 \mathrm{~kg}(\mathrm{H})\end{array}$ \\
\hline Boa chocolate & Epicrates maurus & 2 & $\begin{array}{l}0.6 \mathrm{~kg}(\mathrm{M}) \\
0.6 \mathrm{~kg}(\mathrm{M})\end{array}$ \\
\hline Bejuquillo verde & Leptophis ahaetulla & 1 & $0.08 \mathrm{~kg}(\mathrm{M})$ \\
\hline Bejuquillo café & Oxybelis aeneus & 1 & $0.08 \mathrm{~kg}(\mathrm{H})$ \\
\hline Toche & Spilotes pullatus & 1 & $0.6 \mathrm{~kg}(\mathrm{H})$ \\
\hline Polvo de tabaco & $\begin{array}{c}\text { Thamnodynastes } \\
\text { paraguanae }\end{array}$ & 1 & $0.05 \mathrm{~kg}(\mathrm{M})$ \\
\hline Anaconda & Eunectes murinus & 2 & $\begin{array}{l}12.9 \mathrm{~kg}(\mathrm{M}) ; \\
33.3 \mathrm{~kg}(\mathrm{H})\end{array}$ \\
\hline Total & & 17 & \\
\hline
\end{tabular}

*(M) Macho, (H) Hembra.

Quince serpientes se encontraban bajo cuidados humanos desde hace más de dos años, y dos $B$. constrictor ingresaron a la institución un año antes del inicio de la recolección de las muestras de este estudio, luego de pasar por el proceso de cuarentena descrito a continuación en la sección de medicina preventiva. 
Los ejemplares eran alimentados con ratones (Mus musculus), ratas (Rattus rattus) o pollos (Gallus gallus) criados para este fin en el bioterio de la Fundación Botánica y Zoológica de Barranquilla. La frecuencia de alimentación (semanal o quincenal) y el tipo de presa variaba dependiendo del tamaño de cada individuo.

Las especies criadas en el bioterio eran alimentadas con concentrado comercial y mantenidas en sustrato de aserrín con cambio semanal. Adicionalmente, se realizaban exámenes coprológicos (examen directo y método de Sheather) en pool cada 3 meses a los individuos del bioterio. Se resalta que en la primera semana de recolección de muestras de las serpientes de este estudio, se realizaron los exámenes a los animales del bioterio en los que se encontraron formas parasitarias compatibles con Hymenolepis diminuta y Aspiculuris tetraptera.

Descripción del terrario. Los ejemplares estuvieron alojados en el área de Herpetario, la cual incluye 18 terrarios. Los recintos están elaborados en pared de concreto en tres de sus lados (laterales y fondo), un techo en malla metálica, ventanas con malla metálica (para ventilación) y el frente en vidrio templado para permitir la observación de los animales. Tienen dimensiones aproximadas de $1.1 \times 1.1 \mathrm{~m} \times$ $1.5 \mathrm{~m}$ y cuentan con ambientación compuesta por plantas vivas, ramas secas y diferentes sustratos (arena, piedra china o grava). Debido a las condiciones climáticas de Barranquilla, los terrarios no presentan ningún sistema de calentamiento. El diseño de los recintos no permitía que las serpientes se trasladaran entre terrarios, ni que individuos externos pudieran ingresar a estos, con excepción de insectos como cucarachas o escarabajos. Adicionalmente, iguanas, aves, ardillas y otros roedores de vida libre pueden circular por la parte superior de los terrarios e incluso estar en contacto con la malla ubicada en el techo.

Limpieza y desinfección del terrario. La limpieza y desinfección de los bebederos consistía en la retirada de excretas, lavado de recipientes con agua y jabón diariamente. La desinfección con hipoclorito de sodio al $1 \%$ se realizaba una vez por semana. La desinfección completa de cada terrario se realizaba cuando había traslado de animales a un nuevo recinto o según necesidad por condiciones sanitarias específicas. Esta consistía en el cambio de sustrato y aspersión de glutaraldehído al 0.05\% y amonio cuaternario a $0.01 \%$ en paredes, perchas y sustrato.

Medicina preventiva. Anualmente se realizaba examen clínico a la totalidad de serpientes alojadas en Herpetario. Dicho examen médico consiste en la evaluación de todos los sistemas orgánicos y toma de muestras de sangre (hemograma completo y exámenes bioquímicos) y materia fecal (examen coprológico directo y de flotación). Los animales recibían tratamiento contra parásitos cuando se identificaba un resultado positivo. Nuevos individuos en la colección pasaban por un periodo mínimo de 3 meses en cuarentena aislados de la colección principal. Cada individuo requería tres muestras negativas (examen coprológico directo, de flotación y coloración de Ziehl-neelsen modificado) con un intervalo mínimo de una semana entre la recolección de las muestras y una evaluación clínica antes de ser trasladado al área de Herpetario.

Análisis de las muestras. Se recogieron de 2 a $5 \mathrm{~g}$ de materia fecal del área en que se encontraba el animal. Estas muestras fueron depositadas en frascos plásticos rotulados y almacenadas bajo refrigeración a $4^{\circ} \mathrm{C}$ para su conservación hasta el momento del análisis (tiempo de refrigeración que no superó las $24 \mathrm{~h})$, y buscando recolectar tres muestras por cada animal para realizar el análisis de los datos. Las muestras de ectoparásitos fueron tomadas directamente de la piel del animal y depositadas en tubos eppendorf con alcohol al 70\%. Para la identificación de los endoparásitos, se implementaron los métodos de observación directa y flotación por medio de solución saturada de azúcar descritos por Kassa et al (11). Por otra parte, para identificar los ectoparásitos se utilizó el estereoscopio observando características anatómicas que permitieron la clasificación de los mismos según Quiroz (12). Las prevalencias puntuales reportadas fueron obtenidas dividiendo el número de individuos parasitados sobre el número de individuos evaluados multiplicado por cien.

Finalmente, para los procedimientos de recolección de muestras, manejo y conservación se tuvieron en cuenta las normas éticas, técnicas, científicas y administrativas para la investigación en animales contenidas en la Ley 84 del Congreso de la República de Colombia $y$ el estudio fue realizado por procedimientos de medicina preventiva aprobados por el Departamento de Veterinaria de FUNDAZOO. 


\section{RESULTADOS}

Durante el presente estudio fueron recogidas 42 muestras de materia fecal de un total de 17 individuos (Tabla 2).

El $65 \%(11 / 17)$ de las serpientes muestreadas fueron positivas a la presencia de parásitos; de estos, el 91\% (10/11) fueron boidos. Los nematodos se observaron de forma más frecuente en boidos, mientras que en diapsadinos fueron detectados protozoarios. Se identificó la presencia de protozoarios como flagelados y endoparásitos pertenecientes a la familia Eimeriidae y nematodos como Capillaria sp., huevos larvados y no larvados del orden Strongylida y larvas Rhabditiformes (Figura 1).

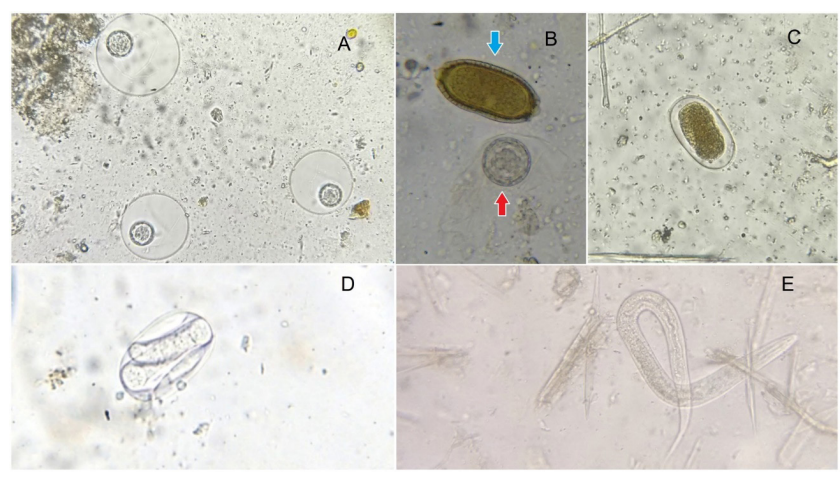

Figura 1. Endoparásitos identificados en las serpientes de FUNDAZOO, objetivo de 400x. A: Ooquiste de parásitos de la familia Eimeriidae hallado en E. murinus, examen directo. B: Huevo de Capillaria sp. (flecha azul) y Ooquiste de parásitos de la familia Eimeriidae (flecha roja) hallado en $E$. murinus, examen directo. C: Huevo no larvado de nematodo del orden Strongylida hallado en $B$. constrictor, examen de flotación. D: Huevo larvado de nematodo del orden Strongylida hallado en B. constrictor, examen flotación. E: Larva Rhabditiforme hallada en $T$. paraguanae, examen directo. Fuente: Duran, 2016.
Dentro de los boidos positivos, los nematodos presentaron mayor prevalencia que los protozoarios (Familia Eimeriidae y Flagelados), identificándose en el $100 \%$ de los boidos $(10 / 10)$ mientras que los protozoarios solo en un $20 \%$ (2/10). En los ejemplares de E. murinus se identificó Capillaria sp. y endoparásitos pertenecientes a la familia Eimeriidae, y por tanto los únicos boidos con parasitosis mixta. En las familias Colubridae y Dipsasidae (Leptophis ahaetulla, Oxybelis aeneus, Spilotes pullatus y Thamnodynastes paraguanae) solo un ejemplar resultó positivo ( $T$. paraguanae), identificándose larvas Rhabdifitormes y flagelados. Los flagelados se identificaron en 3 de las 4 muestras analizadas del individuo de $T$. paraguanae (Tabla 2).

Se determinó una prevalencia de 24\% (4/17) para huevos no larvados de nematodos del orden Strongylida, 18\% (3/17) para huevos larvados de nematodos del orden Strongylida, 12\% (2/17) para parásitos de la familia Eimeriidae y Capillaria sp., $30 \%$ (4/17) para larvas Rhabditiformes y $6 \%$ $(1 / 17)$ para flagelados.

La presencia de parásitos en las muestras positivas varió de la siguiente manera (Tabla 2 ): en $B$. constrictor se identificó un $36 \%$ (4/11) para huevos no larvados de nematodos del orden Strongylida, 27\% (3/11) para huevos larvados de nematodos del orden Strongylida y 9\% (1/11) para larvas Rhabditiformes. En E. murinus se identificó parásitos de la familia Eimeriidae y Capillaria sp. en los dos ejemplares evaluados. Igualmente, en $E$. maurus se identificaron larvas Rhabditiformes en los dos individuos evaluados. Finalmente, de las cuatro muestras recolectas en el único individuo de $T$. paraguanae se identificaron tres muestras positivas para flagelados y una para larvas Rhabditiformes.

Tabla 2. Endoparásitos presentes en muestras de serpientes de FUNDAZOO.

\begin{tabular}{|c|c|c|c|c|c|c|c|c|c|}
\hline \multirow{2}{*}{$\begin{array}{l}\text { Especie (número } \\
\text { de individuos) }\end{array}$} & \multicolumn{6}{|c|}{ Parásitos } & \multirow{2}{*}{$* \mathbf{M}+$} & \multirow{2}{*}{ * M- } & \multirow{2}{*}{ Total } \\
\hline & HNL & HL & Larvas R.* & Familia Eimeriidae & Capillaria sp. & Flagelados & & & \\
\hline B. constrictor (9) & 4 & 3 & 1 & 0 & 0 & 0 & 8 & 11 & 19 \\
\hline E. murinus* $(2)$ & 0 & 0 & 0 & $1 *$ & $1 *$ & 0 & 1 & 0 & 1 \\
\hline E. maurus (2) & 0 & 0 & 3 & 0 & 0 & 0 & 3 & 3 & 6 \\
\hline S. pullatus (1) & 0 & 0 & 0 & 0 & 0 & 0 & 0 & 5 & 5 \\
\hline O. aeneus (1) & 0 & 0 & 0 & 0 & 0 & 0 & 0 & 2 & 2 \\
\hline T. paraguanae (1) & 0 & 0 & 1 & 0 & 0 & 3 & 4 & 3 & 7 \\
\hline L. ahaetulla (1) & 0 & 0 & 0 & 0 & 0 & 0 & 0 & 2 & 2 \\
\hline Total & 4 & 3 & 5 & $1 *$ & $1 *$ & 3 & 16 & 26 & 42 \\
\hline
\end{tabular}

$\mathrm{HNL}=$ Huevo no larvado del orden Strongylida; $\mathrm{HL}=$ Huevo larvado del orden Strongylida *La muestra de $E$. murinus fue recolectada en pool; Larvas R: Larvas Rhabditiformes; *M+: Muestras positivas; *M-: Muestras negativas 
Además de lo anterior, durante el estudio se obtuvieron siete muestras de ectoparásitos pertenecientes: a 5 terrarios de Boa constrictor, al único ejemplar de Spilotes pullatus y a un ejemplar de Epicrates maurus (Tabla 3). En seis de las muestras de ectoparásitos se identificó la garrapata Ornithodoros turicata (Figura 2) perteneciente a la familia Argasidae. En una de las muestras perteneciente a $B$. contrictor se identificó la garrapata Amblyomma dissimile (Figura 3) perteneciente a la familia Ixodidae.

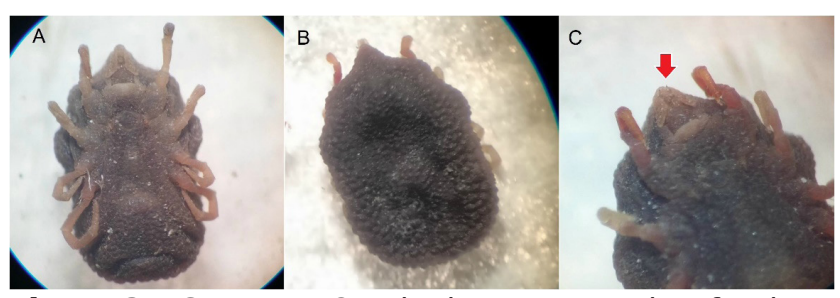

Figura 2. Garrapata Ornithodoros turicata identificada en B. constrictor, S. pullatus y E. maurus. A. vista ventral, B. vista dorsal (cuerpo mamelonado) y C. hipostoma poco desarrollado (flecha roja). Fuente: Duran, 2016.

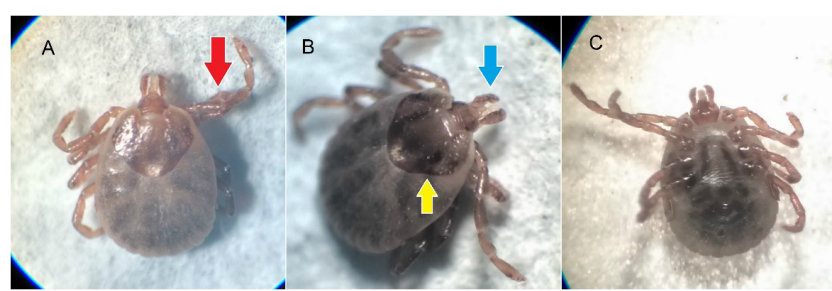

Figura 3. Garrapata Amblyomma dissimile identificada en $B$. constrictor, palpos largos (flecha roja) con el segundo segmento menos largo que ancho. En la imagen se observa: A. vista dorsal, B. escudo (flecha amarilla) e hipostoma (flecha azul) y C. vista ventral. Fuente: Duran, 2016.

Tabla 3. Ectoparásitos presentes en muestras de serpientes de FUNDAZOO.

\begin{tabular}{ccc}
\hline \multirow{2}{*}{ Especie } & \multicolumn{2}{c}{ Ectoparásitos } \\
\cline { 2 - 3 } & A. dissimile & O. turicata \\
\hline Boa constrictor & 1 & 4 \\
Eunectes murinus & 0 & 0 \\
Epicrates maurus & 0 & 1 \\
Spilotes pullatus & 0 & 1 \\
Oxybelis aeneus & 0 & 0 \\
Thamnodynastes & 0 & 0 \\
paraguanae & 0 & 0 \\
Leptophis ahaetulla & $\mathbf{1}$ & $\mathbf{6}$ \\
\hline Total & &
\end{tabular}

Los ectoparásitos anteriormente nombrados presentaron mayor prevalencia en los boidos que en los colúbridos, ya que solo un colúbrido
(S. pullatus) resultó positivo a la presencia de ectoparásitos: Sin embargo, se debe tener en cuenta que el número de boidos fue superior al de colúbridos. La especie de garrapata que presentó mayor prevalencia fue Ornithodoros turicata, encontrándose en seis ejemplares de $B$. constrictor y en un individuo de $S$. pullatus mientras que Amblyomma dissimile solo se identificó en un ejemplar de $B$. constrictor como se mencionó anteriormente.

\section{DISCUSIÓN}

En el presente estudio el 65\% (11/17) de los animales fue positivo a la presencia de parásitos gastrointestinales tal como se describe en la tabla 2. Se identificaron 2 especies de protozoarios (Flagelados y parásitos de la Familia Eimeriidae) y 4 tipos de nematodos (Huevo no larvado y huevos larvados de nematodo del orden Strongylidae, Capillaria sp. y larvas Rhabditiformes). En el 27\% (3/11) de los individuos se presentó parasitosis mixta y en el $73 \%$ (8/11) solo parasitosis por nematodos (Tabla 2 ).

La mayor prevalencia de nematodos de ciclo de vida directo se ve atribuida a la característica de estos de presentar formas parasitarias de vida libre, lo cual les permite sobrevivir en el medio ambiente largas temporadas, esperando el momento indicado para afectar el hospedador, lo que favorece la reinfección (12). Por otra parte, los boidos (Boa constrictor, E. maurus y E. murinus) representaron el 91\% (10/11) de los ejemplares positivos, en los cuales se identificaron ambos filos parasitarios (protozoarios y nematodos). Sin embargo, se debe tener en cuenta que son el grupo taxonómico con mayor número de individuos en el presente estudio.

Los ejemplares fueron asintomáticos a las descripciones clínicas características de nematodos y protozoarios como lo son: anorexia, letargia, regurgitación postprandial, obstrucción, lesiones vasculares, dificultad respiratoria, pérdida de peso, enteritis aguda y diarrea, $(5,13,14)$. Sin embargo, el único individuo de polvo de tabaco ( $T$. paraguanae), presentó heces acuosas y mucosas durante el estudio, uno de los síntomas atribuidos a parasitosis por flagelados (15).

Los resultados concuerdan con literatura en relación con la identificación de formas parasitarias (14), sin embargo, parásitos de la familia Eimeriidae son reportados como 
el género de coccidia de mayor incidencia en reptiles ex situ, mientras que en este estudio la prevalencia fue baja (12\%). Otros autores $(11,13,15,16,17)$, reportaron la identificación de nematodos (Strongylus sp. y Strongyloides sp.), Capillaria sp., flagelados y Eimeria sp., en serpientes; resultados que coinciden con los del presente estudio. Protozoarios flagelados son reportados como la fauna parasitaria mayormente encontrada en serpientes ex situ (18). No obstante, esto difiere con los resultados obtenidos en este trabajo, ya que solo un ejemplar resultó positivo a parasitosis por flagelados. Por otra parte, la presencia de parasitosis mixta fue mayoritaria a la parasitosis simple concordando con un estudio realizado por Nasiri et al (19).

Algunos autores han encontrado el parásito Hymenolepis spp. en muestras de serpientes y los han clasificado como pseudoparásitos, ya que provienen de roedores infectados sin ser patogénicos para serpientes $(3,20)$. Sin embargo, esta relación no se presentó en esta investigación ya que, aunque las fuentes de alimentación (ratas y ratones y pollos) estuvieran contaminadas (Hymenolepis diminuta y Aspiculuris tetraptera), estas formas parasitarias no fueron identificadas en los coprológicos realizados en este estudio.

Con relación a los ectoparásitos, se identificó la garrapata Amblyomma dissimile en un ejemplar de $B$. constrictor, del mismo modo como es reportado en literatura $(9,10)$. Es importante resaltar esta especie como transmisora de Hepatozoon sp. y Rickettsia sp. y que esta afecta a especies como Epicrates spp. $(7,8)$. Aunque $A$. dissimile solo afectó a uno de los 17 individuos del estudio, no se puede descartar su presencia en un futuro, ya que es posible que se encuentre contaminando el sustrato u otras partes de la exhibición. Además de esto, el parásito ha sido reportado como la garrapata que más afecta a reptiles ex situ en América Central y América del Sur (8).

Al igual que los anteriores, algunos autores reportan $A$. dissimile como la garrapata que más afecta a serpientes de la especie $B$. constrictor (21). Asimismo, otros reportan hallazgos de especies de Ammblyoma sp. en E. murinus (22), sin embargo, a diferencia de estos reportes, E. murinus no presentó ectoparásitos en el presente estudio. Finalmente, en contraste con el reporte de afección de $A$. dissimile en colúbridos (23), este ectoparásito no se presentó en los colúbridos evaluados en esta investigación.
Adicional a la especie de garrapata anterior, se identificó Ornithodoros turicata, la cual ya ha sido reportada en otros países afectando a reptiles (24). De acuerdo con nuestro conocimiento, este es el primer reporte de dicha especie de garrapata cómo ectoparásito en serpientes en Colombia. Asimismo, se da a conocer que esta garrapata, originaria de Estados Unidos y México, es también reconocida como un vector de la espiroqueta (Borrelia turicatae) causante de fiebre recidivante y posible transmisor del virus de la peste porcina africana (24). O. turicata solo se encontró en muestras de seis boidos ( $B$. constrictor y $E$. maurus) y solo un colúbrido ( $S$. pullatus).

Con relación a la posibilidad de transmisión de virus, cabe resaltar que un ejemplar de $B$. constrictor fallece en la octava semana del estudio con sintomatología nerviosa. Posiblemente, la muerte podría estar asociada a la enfermedad del corpúsculo de inclusión (IBD, inclusion body disease, sigla en inglés) luego de presentar una infestación por esta especie de garrapata. Aunque este diagnóstico no se logró confirmar debido a que el espécimen presentó alto grado de autolisis que no permitieron la toma de muestras para histopatología, es posible que haya relación entre esta especie de garrapata y el retrovirus causal de la IBD.

En conclusión, la variedad de formas endoparasitarias y ectoparasitarias coincide con los reportes de literatura. Sin embargo, la prevalencia en este estudio difirió de los mismos teniendo en cuenta que fue desarrollado con animales bajo cuidados humanos, lo que constituye un factor importante a considerar. Las fuentes de alimentación y agua de cada una de las especies estudiadas cuentan con planes de vermifugación, limpieza y desinfección que disminuyen la probabilidad de ocurrencia de parásitos internos contraídos por alimentación. Además sus terrarios cuentan con un plan de limpieza y desinfección que disminuye notablemente la presencia de ectoparásitos.

Por otro lado, existen iguanas (Iguana iguana) libres dentro de las instalaciones del zoológico las cuales pueden ser una fuente de transmisión de parásitos. Considerando que se encontraron solamente parásitos de ciclo directo y que todas las serpientes salieron del periodo de cuarentena negativas a parásitos, es probable que estos ejemplares se hayan contaminado por el contacto con excretas de las iguanas que circulan por el área o por insectos cómo hospedadores paraténicos. 
Finalmente, la identificación de las formas parasitarias en ofidios en este trabajo es una contribución importante para la parasitología de este grupo de reptiles en Colombia, debido a que provee información para desarrollar medidas profilácticas y permite comparar los datos sobre las formas parasitarias encontradas con muestreos de estas especies en vida silvestre y ex situ.

\section{Conflicto de intereses}

Los autores del presente artículo manifiestan no tener ningún tipo de conflicto de intereses relacionado con la parte financiera, intelectual, familiar o de pertenencia que constituyan un conflicto, ya sea efectivo, potencial o aparente.

\section{Agradecimientos}

A Fundación Botánica y Zoológica de Barranquilla "Barranquilla Zoo" por permitir el desarrollo del estudio en conjunto con la Universidad de Pamplona.

\section{REFERENCIAS}

1. Mellish S, Ryan J.C, Pearson E.L, Tuckey M.R. Research methods and reporting practices in zoo and aquarium conservation-education evaluation. Conserv Biol. 2019; 33(1):4052. https://doi.org/10.1111/cobi.13177 _

2. Bais B, Tak L, Mahla S. Study of preventive health measures for wildlife in captivity: a review of management approaches. Int J Avian \& Wildlife Biol. 2017; 2(3):73-75. https://doi.org/10.15406/ ijawb.2017.02.00020

3. Okulewicz A, Kaźmierczak M, Zdrzalik K. Endoparasites of exotic snakes (Ophidia). Helminthologia. 2014; 51(1):31-36. https:// doi.org/10.2478/s11687-014-0205-z

4. Villalobos F, Troncoso I, Loyola E, Robles A, Aguilar J, Fernández I et al. Determinación coproscópica de formas parasitarias en heces de ofidios: Boa constrictor y Python regius. Revista Científica FCV-LUZ. 2014; 24(5):454-457. URL Disponible en: http:// produccioncientificaluz.org/index.php/ cientifica/article/view/11805 .

5. González D, Durán J, Cedeño J. Helmintos parásitos de Boa constrictor (Serpentes: Boidae) en el sur de Quintana Roo. Rev Mex Biodivers. 2014; 85(3):831-837. https:// doi.org/10.7550/rmb.40515
6. Wolf D, Globokar-Vrhovec M, Failing K, Rossier C, Hermosilla C, Pantchev N. Diagnosis of gastrointestinal parasites in reptiles: comparison of two coprological methods. Acta Vet Scand. 2014; 56(44): 1-13. https://doi.org/10.1186/s13028-0140044-4

7. Chávez L, Serrano-Martínez H, Tantaleán M, Quispe M, Casas G.C. Parásitos Gastrointestinales en Reptiles en Cautiverio en Lima Metropolitana. Rev Inv Vet Perú. 2015; 26(1):127-134. http://dx.doi. org/10.15381/rivep.v26i1.10909

8. Novakova M, Literak I, Chevez L, Martins F, Ogrzewalska M, Labruna B. Rickettsial infections in ticks from reptiles, birds and humans in Honduras. Ticks Tick Borne Dis. 2015; 6(6):737-742. https://doi. org/10.1016/j.ttbdis.2015.06.009

9. Carrascal J, Oviedo T, Monsalve S, Torres A. Amblyomma dissimile (Acari: Ixodidae) parásito de Boa constrictor en Colombia. Rev MVZ Córdoba. 2009; 14(2):1745-1749. https://doi.org/10.21897/rmvz.358

10. Paternina L, Verbel E, Bejarano E. First report of Amblyomma dissimile (Acari: Ixodidae) on Spilotes pullatus (Squamata: Colubridae) from Colombia. Rev Inv Med Trop. 2015; 1(1):23-25. URL Disponible en: https://repositorio.unisucre.edu.co/ 


\section{$\underline{\text { handle/001/968 }}$}

11. Kassa D, Mengestie A, Ayalew N, Belaynew A, Mebrie Z, Metadel T. A Review on Diagnostic Techniques in Veterinary Helminthlogy. Nat Sci. 2016; 14(7):109-118. https://doi. org/10.7537/marsnsj140716.15

12. Quiroz H. Parasitología. 4th. ed. Mexico D.F: LIMUSA S.A; 1990.

13. Md-Robiul $K$, Fuchang $Y$, Jian $L$, Junqiang $L$, Longxian $Z$, Rongjun W et al. First molecular characterization of enteric protozoa and the human pathogenic microsporidian, Enterocytozoon bieneusi, in captive snakes in China. Parasitol Res. 2014; 113(8):30413048. https://doi.org/10.1007/s00436014-3967-9

14. Vergles A, Lindtner-Knific R, Vlahović K, Mavri $U$, Dovč A. Parasites in pet reptiles. Acta Vet Scand, 2011; 53(1):1-20. https:// doi.org/10.1186/1751-0147-53-33 _

15. Jepson, L. Medicina de animales exóticos guía de referencia rápida. $1^{a}$. Ed. España: Elsevier; 2011.

16. Rinaldi L, Mihalca A, Cirillo R, Maurelli M, Montesano M, Capasso $M$ et al. Flotac can detect parasitic and pseudoparasitic elements in reptiles. Exp Parasitol. 2012; 130(3):282-284. https://doi.org/10.1016/j. exppara.2012.01.011

17. Panayotova M. Parasites in captive animals: a review of studies in some european zoos. Zol Gart. 2013; 82(1-2):60-71. https://doi. org/10.1016/j.zoolgart.2013.04.005

18. Scullion F, Scullion M. Gastrointestinal protozoal diseases in reptiles. J Exot Pet Med. 2009; 18(4):266-278. https://doi.

\section{org/10.1053/j.jepm.2009.09.004}

19. Nasiri V, Mobedi I, Dalimi A, Zare A, Ghaffarifar F, Teymurzadeh $S$ et al. A description of parasites from Iranian snakes. Exp Parasitol. 2014; 147:7-15. https://doi. org/10.1016/j.exppara.2014.09.007

20. Raś-Noryńska M, Sokół R. Internal parasites of reptiles. Ann Parasitol. 2015; 61(2):115117. https://www.annals-parasitology. eu/go.live.php/download default/D667/ internal-parasites-of-reptiles.html

21. Mogollón M, Pineda A, Benavides $Y$, Vargas L. Amblyomma dissimile en Boa constrictor en cautiverio del Centro de Atención y Valoración de Fauna Silvestre de Montería (Córdoba, Colombia). Rev Med Vet. 2017; (35): 29-34. http://dx.doi.org/10.19052/ $\underline{\mathrm{mv} .4386}$

22. Guerrero R, Rodríguez-Acosta A. Primer reporte de infestación de la serpiente reinita Erythrolamprus melanotus (Shaw, 1802) (Serpentes: Dipsadidae) por el ácaro Amblyomma dissimile Koch, 1844 (Acarina:Ixodidae) en Venezuela. Cuad Herpetol. 2014; 28(2):241-143. URL disponible en: http://ppct.caicyt.gov.ar/ index.php/cuadherpetol/article/view/3052

23. Mihalca A. Ticks imported to Europe with exotic reptiles. Vet Parasitol. 2015; 213(12):67-71. https://doi.org/10.1016/j. vetpar.2015.03.024

24. Donaldson, T.G, Pèrez de León A, Li $A$, Castro-Arellano I, Wozniak W, Boyle W et al. Assessment of the Geographic Distribution of Ornithodoros turicata (Argasidae): Climate Variation and Host Diversity. PLOS Neglected Tropical Diseases. 2016; 10(3):e0004383. https://doi.org/10.1371/ journal.pntd.0004383 